\title{
Dynamique de l'involution utérine et de la croissance folliculaire pendant l'anoestrus postpartum chez le zébu peulh d'Afrique
}

\author{
Auguste Tanguy YAMBOUE ${ }^{1}$, Moussa $\mathrm{ZONGO}^{1 *}$, Boureima TRAORE ${ }^{1}$ et \\ Boureima Harouna KARIMOU ${ }^{2}$ \\ ${ }^{1}$ Laboratoire de Physiologie Animale, UFR /SVT, Université Joseph KI - ZERBO, 03 BP 7021 Ouagadougou \\ 03, Burkina Faso. \\ ${ }^{2}$ Faculté des Sciences d'Agronomie, Université de Tillabéri, BP 175 - Niger. \\ *Auteur correspondant ; E-mail : moussa_zongo59@yahoo.fr, Tel : (00226) 70259047
}

\section{REMERCIEMENTS}

Les auteurs remercient la Fondation International pour la Science (FIS) pour leur appui technique et financier dans la réalisation de la présente étude. Ils souhaitent en outre, que l'Agence Internationale pour l'Energie Atomique (AIEA) trouve à travers cet article, l'expression de leur reconnaissance pour les équipements offerts.

\section{RESUME}

Les perspectives d'une remise en reproduction après vêlage, prennent en compte la durée de l'anoestrus postpartum. Cette durée dépendrait du délai d'involution utérine et du fonctionnement de l'axe gonadohypothalamo-hypophysaire. L'objectif de cette étude a été d'apprécier les caractéristiques de l'anoestrus postpartum, notamment l'involution utérine complète et la reprise de l'activité ovarienne après la parturition chez la vache zébu Peuhl. Elle a concerné dix femelles $(\mathrm{n}=10)$ d'âge et de poids moyens compris respectivement dans les intervalles [4 - 13] ans et [190 - 250] kg. Elles ont été examinées à partir deJ7 jusqu'à J100 postpartum, au moyen d'un échographe portable muni d'une sonde linéaire de 7,5 Mhz. Les clichés les plus représentatifs des cornes utérines, du cervix et des organites ovariens ont été enregistrés et analysés. La courbe de résorption utérine a montré une phase de régression rapide entre J7 et J16 suivi d'une phase lente entre J17 et J45.Ledélai moyen pour l'involution utérine a été $26,6 \pm 2,11$ jours. La reprise de l'activité ovarienne a été observée dans un délai de $68,5 \pm 6,11$ jours. Ce délai varie en fonction de la parité $(p=0,04)$.

(c) 2020 International Formulae Group. All rights reserved.

Mots clés : Involution utérine, activité ovarienne, postpartum, zébu.

\section{Dynamics of uterine involution and follicular growth during anoestrus postpartum in African zebu Peulh}

\begin{abstract}
The prospects for rebreeding after calving were strongly influenced by postpartum anestrus duration which depends on the time of complete uterine involution and ovarian activity resumption. The aim of this study was to assess the characteristics of postpartum anestrus, in particular complete uterine involution and resumption
\end{abstract}


of ovarian activity in the Peuhl zebu. It carried out on ten females $(n=10)$ which mean age and weight were extended between [4 - 13] years and [190 - 250] kg respectively. They were examined from Day 7 to Day 100 after calving by using a portable ultrasound scanner equipped with a $7.5 \mathrm{Mhz}$ linear probe. The most representative images of uterine horns, cervix and ovarian structures have been frozen, recorded and exported for analyzing. The uterine resorption curve showed a rapid regression phase between Day 7 to 16 followed by a slow phase between D17 and D45. The mean time for uterine involution was $26.6 \pm 2.11$ days. Resumption of ovarian activity was observed within $68.5 \pm 6.11$ days. This time varies with parity $(\mathrm{p}=0.04)$.

(C) 2020 International Formulae Group. All rights reserved.

Keywords: Uterine involution, ovarian activity, postpartum, Zebu cows

\section{INTRODUCTION}

Au Burkina Faso, l'élevage contribue entre 18,3 et 19,5\% au Produit Intérieur Brute PIB (MRA, 2011) et 26\% aux recettes d'exportation, après le coton (MRA, 2010). Cette contribution au plan macro-économique représente sans conteste, des recettes propres dans la mesure où ce secteur qui est encore largement extensif, est très peu dépendant de l'approvisionnement en intrants importés (FAO, 2018).'élevage du zébu est celui pratiqué par la plupart des ménages du pays et participe fortement à la résolution des problèmes socio-culturels et économiques des communautés pastorales sahéliennes (traction animale, transport et apport de fumure). A côté de son apport important à l'économie, il contribue également de façon efficace à la sécurité alimentaire et nutritionnelle des populations, particulièrement en milieu rural où près de $86 \%$ de la population en tire entièrement ou partiellement ses revenus (FAO, 2018).

L'élevage du zébu est pourtant, confronté au problème de développement et d'amélioration des pouvoirs d'achat des producteurs. Il a conservé un caractère traditionnel caractérisé par une conduite extensive des troupeaux sur le pâturage naturel (Zampaligré et al., 2019). Très adapté à son habitat naturel des tropiques, le zébu présente de faibles potentialités de production (Pitala et al., 2012 ; Mwaï et al., 2015 ; Seme et al., 2017). Mais, eu égard aux frais minimes occasionnés pour leur suivi, la rentabilité est néanmoins réelle. Les performances de reproduction ne sont guère meilleures. Les proportions d'anoestrus postpartum sont élevées et la fertilité après la parturition est faible (Abeygunawardena et Dematawewa, 2004 ; Marichatou et al., 2010). Ce problème contribue à ladégradation de l'environnement socio-économique des éleveurs.

Les données sur la dynamique des follicules ovariens et les caractéristiques de l'involution utérine au cours du postpartum chez le zébu Peuhl sont peu documentées. Contrairement aux zébus Azawak et Goudali, le zébu Peuhl présente des exigences fourragères moins élevées, s'engraisse facilement et résiste mieux aux maladies transmises par les tiques (Touré et al., 2019 ; Yahaya et al., 2019).

La présente étude avait pour objet d'apprécier les caractéristiques de l'anoestrus postpartum chez la femelle zébu en milieu soudano-sahélien. Il s'est agi d'apprécier par observation échographique la dynamique de l'involution utérine complète et l'ovulation après la parturition chez le zébu.

\section{MATERIEL ET METHODES \\ Animaux et traitement}

Cette étude a concerné dix femelles parturientes $(n=10)$ de race zébu peuhl, d'âge et de poids moyen compris respectivement dans les intervalles [4 -13] ans et [190 - 250] $\mathrm{kg}$. Elles ont été réparties en deux lots en fonction du numéro de vêlage. Le lot 1 a été composé de femelles primipares $(n=5)$ et le lot 2 , de femelles multipares $(n=5)$. Les veaux ont été laissés avec leur mère au pâturage comme à 
l'étable. La ration a été complétée au retour du pâturage par des tourteaux de coton ou des drêches de brasseries à raison de $1 \mathrm{~kg} /$ femelle et de blocs minéraux (pierre à lécher) en permanence. L'eau a été disponible à volonté. Le programme national de prophylaxie contre les grandes épizooties (fièvre aphteuse, brucellose, péripneumonie contagieuse, etc) a été régulièrement suivi.

\section{Examens échographiques de l'utérus et des ovaires}

Les examens de l'utérus et des ovaires ont été réalisés par voie transrectale au moyen d'un échographe Easi-scan (IMV Imaging, BCF Technology) équipé d'une sonde à balayage linéaire de 7,5 Mhz. Les observations ont été pratiquées à intervalle d'un jour sur deux par le même opérateur. Pour des raisons pratiques, les examens des caroncules utérines ont débuté à partir de $\mathrm{J} 3$ et ceux des diamètres utérins à partir de $\mathrm{J} 7$ jusqu'à la fin de l'involution utérine compète et l'apparition d'une deuxième ovulation. Les images échographiques correspondant aux sections transversales du cervix comme illustré sur la Figure $1 \mathrm{~B}$ et des cornes utérines (Figure $1 \mathrm{C}$ ) ont été obtenues selon le protocole décrit par Heppelmann et al. (2013) et Canadas et al. (2019). Les critères d'une involution utérine complète ont été la tendance à la symétrisation des cornes utérines ou à la faible variation entre les dimensions des deux cornes utérines au cours de quatre mesures successives comme présenté à la Figure $1 \mathrm{D}$, l'absence de lochies et la stabilisation du col utérin comme illustré à la Figure 1E. Les examens échographiques des ovaires ont été réalisés suivant le protocole décrit par Pierson et Ginther (1988) et Savio et al. (1990). Chaque ovaire a été saisi au moyen de l'index et du majeur, puis accolé à la sonde afin de visualiser les structures ovariennes. Les paramètres évalués ont été la taille et le nombre de follicules par ovaire, les délais manifestations d'œstrus et des ovulations et après parturition. L'ovulation a été défini comme la disparition du follicule dominant entre deux observations consécutives suivie du développement d'un corps jaune dans la position précédemment occupée par le follicule ovulatoire (Savio et al., 1990).

\section{Détections des oestrus}

La détection de l'œstrus a été réalisée deux fois par jour (matin et soir), par observations visuelles directes, des comportements des femelles, en présence de deux taureaux fertiles de bonne libido. Ces géniteurs ont été introduits dans l'étable expérimentale durant 30-45 minutes en présence des techniciens. Le critère fondamental d'un œstrus vrai est le chevauchement. D'autres critères tel que, l'écoulement de glaire, la tumescence de la vulve, le reniflement du taureaux, les montes passives et les beuglements sont des critères complémentaires de l'oestrus (Toribo et al., 1995 ; Sakaguchi et al., 2004). Une palpation manuelle transrectale de l'appareil génital (ovaires et tractus) a permi de confirmer les observations.

\section{Analyse statistique}

Les résultats recueillis ont été exprimés en moyenne \pm écart-type et analysés au moyen du logiciel R (version 3.5.1, version Windows). Les variations des délais de l'involution utérine complète et de l'activité ovarienne selon la parité ont été appréciées avec le test de Mann Whitney. Les variations des diamètres des follicules, de l'utérus (cornes utérines et cervix) et des caroncules utérines ont été évaluées avec le test $t$ de Student. Les résultats ont été considérés comme significatifs au seuil de probabilité de $5 \%$. 


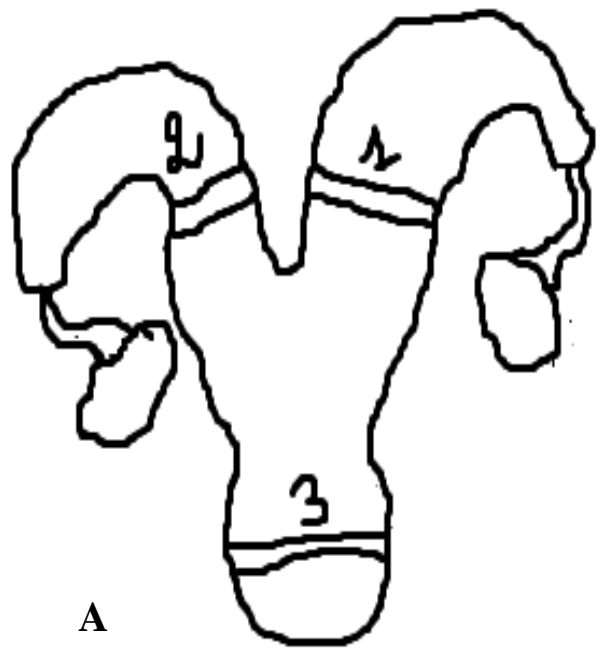

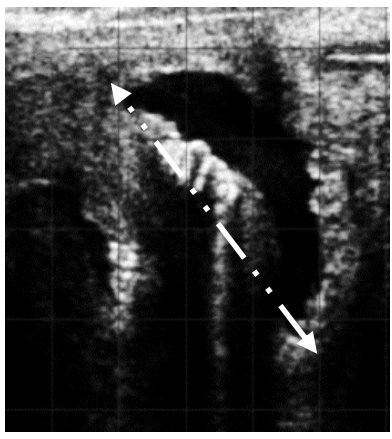

B. Mesure échographique du diamètre cervical

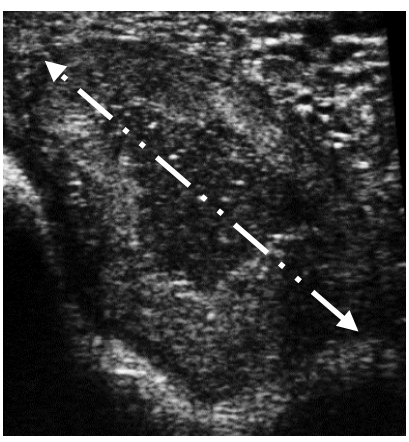

C. Mesure échographique du diamètre de la corne utérine

(1) Diamètre de la corne précédemment gravide ; (2) Diamètre de la corne non gravide ;

3) Diamètre du cervix ou col de l'utérus.

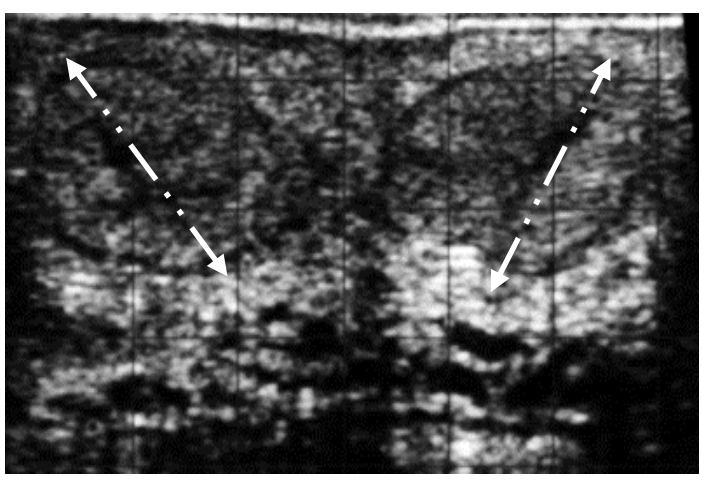

D. Symétrisation des cornes utérines

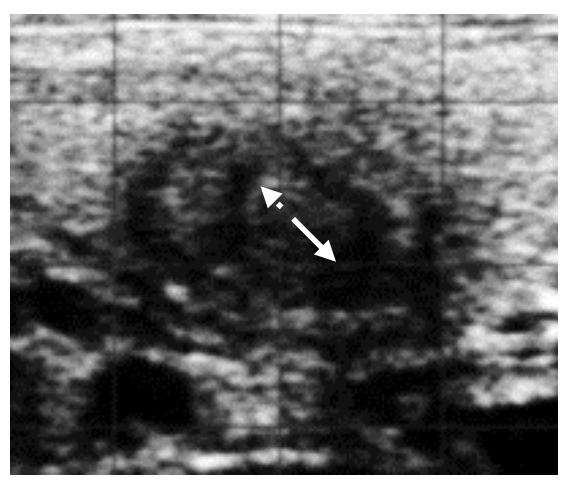

E. résorption du col de l'utérus

Figure 1: Illustration des dispositions de la sonde pour les mensurations et des images échographiques du diamètre des cornes utérines et du col de l'utérus au cours du postpartum chez le zébu.

\section{RESULTATS \\ Involution utérine}

Chez la femelle zébu Peulh à J3 postpartum, la lumière de l'utérus est anéchogène avec la présence de caroncules sur la paroi de l'utérus (Figure 2A). Les limites (diamètre et longueur) de la corne utérine à cette date ne sont pas suffisamment nettes, rendant les mesures échographiques approximatives (Figure 2B).

Les caroncules utérines sous forme de nodules échogènes ont régressé de $10,7 \mathrm{~mm}$ en moyenne entre J3 et J7 (Figure 2 ; Figure 3). Durant cette période, aucune variation significative n'a été constatée entre femelles primipares et multipares ( $p>0,05)$ (Tableau 1 ). Le contenu de la corne utérine s'est progressivement éclairci entre $\mathrm{J} 4$ à $\mathrm{J} 6$ et le diamètre mesurable à partir de $\mathrm{J} 7(79,06 \pm 7,54$ mm) (Figure 3).

Le nombre de caroncules utérines observables à l'échographie a connu une forte réduction entre $\mathrm{J} 9$ et $\mathrm{J} 12$.Les diamètres moyens des caroncules utérines ont varié en fonction de la parité (Tableau 1). La disparition complète des lochies a été constatée entre J12 et J18 pp. Les images échographiques de l'involution du cervix ont été rapportées à la Figure 4. Entre 
J3 et J7 pp le cervix n'est observable, la distension du cervix pendant de cette période est telle que les limites ne sont pas perceptibles à l'échographie. Il réapparait progressivement à partir de $\mathrm{J} 9$ et le diamètre devient mesurable, individualisé à J14.

Les diamètres des cornes utérines précédemment gestantes ont été plus grands que ceux des cornes non gestantes entre $\mathrm{J} 7$ et J12 pp (Figure 5A). Cette variation a montré des différences significatives (Figure 5A) entre $\mathrm{J} 7$ et J12 (Figure 5A). A partir de J16 les courbes montrant les involutions utérines de la corne précédemment gestante et de la corne précédemment non gestante se sont supperposées (Figure 5A). L'analyse de ces deux courbes montre une régression utérine en deux phases. Une phase rapide d'involution utérine pour les cornes précédemment gestante et non gestante entre $\mathrm{J} 7$ et $\mathrm{J} 16(5,71 \pm 1,88 \mathrm{~mm}$ /jour, 4,44 $\pm 1,37 \mathrm{~mm} /$ jour, respectivement) et une phase lente entre $\mathrm{J} 17$ et $\mathrm{J} 45(0,75 \pm 0,38$ vs $0,7 \pm 0,19 \mathrm{~mm} /$ jour, respectivement $)(p>0,05)$ (Figure 5A). Le délai moyen pour l'involution utérine a été de 26,6 $\pm 2,11$ jours. Ce délai varie en fonction de la parité mais reste cependant non significatif ( $p>0,05$; Tableau 2). Le diamètre moyen des cornes utérines après résorption complète a été de 17,19 $\pm 1,81 \mathrm{~mm}$.

La résorption cervicale a été plus lente comparativement aux cornes utérines $(\mathrm{p}=$ 0,01). L'analyse de la courbe (Figure 5B) montre une régression cervicale en deux phases. Une phase rapide entre J7 et J33 (1,11 $\pm 0,55 \mathrm{~mm} /$ jour) et une phase lente entre $\mathrm{J} 34$ et J47 $(0,4 \pm 0,43 \mathrm{~mm} /$ jour $)$.

Le délai moyen de l'involution du cervix utérin a été de $37,5 \pm 5,01$ jours. Ce délai est par contre plus tardif chez les femelles multipares comparativement aux femelles primipares, mais sans différences significatives ( $>$ > 0,05; Tableau 2). En outre, les vitesses de résorption utérine et du cervix sont variables entre femelles multipares et primipare (Tableau 2).

\section{Evolution de structures ovariennes et reprise de la cyclicité}

A J7 pp les examens échographiques ont rapporté des images de structures sur les deux ovaires. Des corps jaunes atrétiques
(Figure 6) de diamètre moyen 8,43 $\pm 1,48 \mathrm{~mm}$ ont été observés sur les ovaires de 7 femelles (Figure 6a). Ces corps jaunes ont été examinés sur les ovaires ipsilatéraux aux cornes précédemment gestantes. A J9 pp, ces corps jaunes ont subi une régression pour progressivement disparaître entre J10 et J15 postpartum.

A J7 pp, des follicules dont le nombre varie entre 2 et 4 avec un diamètre moyen de 4,69 $\pm 1,17 \mathrm{~mm}$ ont en outre été observés chez 9 femelles (Tableau 3). Deux follicules de diamètres $8,75 \mathrm{~mm}$ et $9,37 \mathrm{~mm}$ ont été rapportés chez une femelle à $\mathrm{J} 7 \mathrm{pp}$. Le nombre des follicules sur les ovaires gauches et droits ont été respectivement $3 \pm 1,06$ et 2,75 $\pm 0,95$ follicules. A cette date, il n'a pas été observé de variations significatives du nombre, ni de la taille des follicules entre les deux ovaires. La taille des follicules a augmenté au cours des observations successives pour atteindre des valeurs maximales de $10,41 \pm 2,60 \mathrm{~mm}$ à $\mathrm{J} 12$ et J13 chez l'ensemble des vaches. Ces follicules ont disparu à partir de J14. Aucune modification comportementale n'a été observée chez les femelles à cette date. Entre $\mathrm{J} 14$ et $\mathrm{J} 30$, des follicules de diamètre variant entre 3 et $5 \mathrm{~mm}$ ont été observés. Les premières manifestations d'œstrus accompagnées d'ovulations au cours du postpartum ont été observées à J55 et J67 successivement chez deux femelles multipares. La taille moyenne des follicules à l'ovulation a été $10,31 \pm 1,32$ $\mathrm{mm}$. Les manifestations d'œstrus observées ont été les écoulements de glaires et les chevauchements. Les huit autres femelles zébu $(\mathrm{n}=8 / 10)$ ont présenté des premières ovulations non précédées d'œstrus. Le délai moyen de la première ovulation après la parturition a été de $70 \pm 5,15$ jours et cette durée a varié en fonction de la parité (Tableau 3). Les femelles multipares semblent avoir des délais de premières ovulations significativement précoces comparativement aux femelles primipares $(p=0,04)$ (Tableau 3$)$. Le délai moyen de la deuxième ovulation a été $88,5 \pm 4,45$ jours. A cette deuxième ovulation, l'œstrus a été observé chez six femelles (4 multipares et 2 primipares). La taille moyenne des follicules à la deuxième ovulation a été de $11,04 \pm 1,57 \mathrm{~mm}$. 


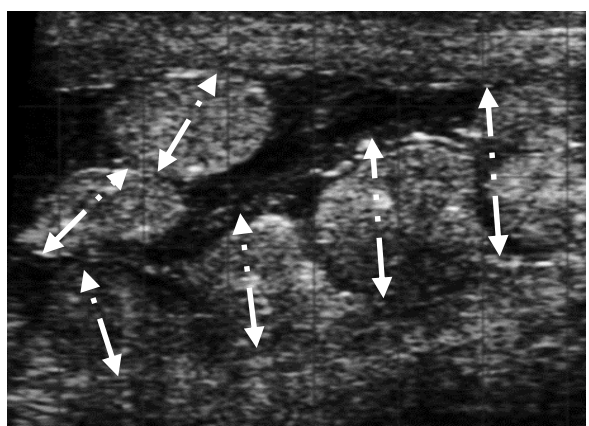

A. Coupe longitudinale de la corne utérine

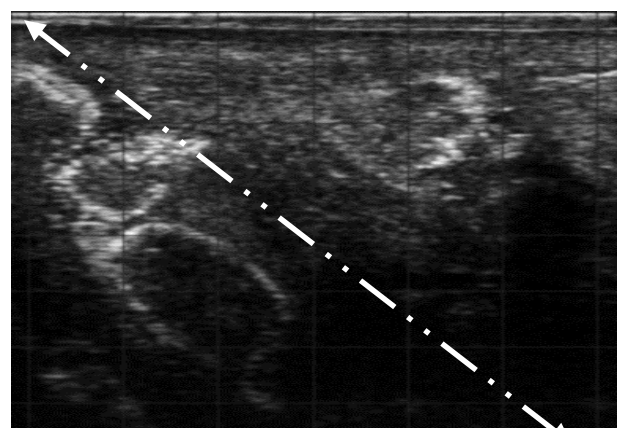

B. Coupe transversale de la corne utérine

Figure 2 : Images échographiques de la corne utérine à J3 près parturition.
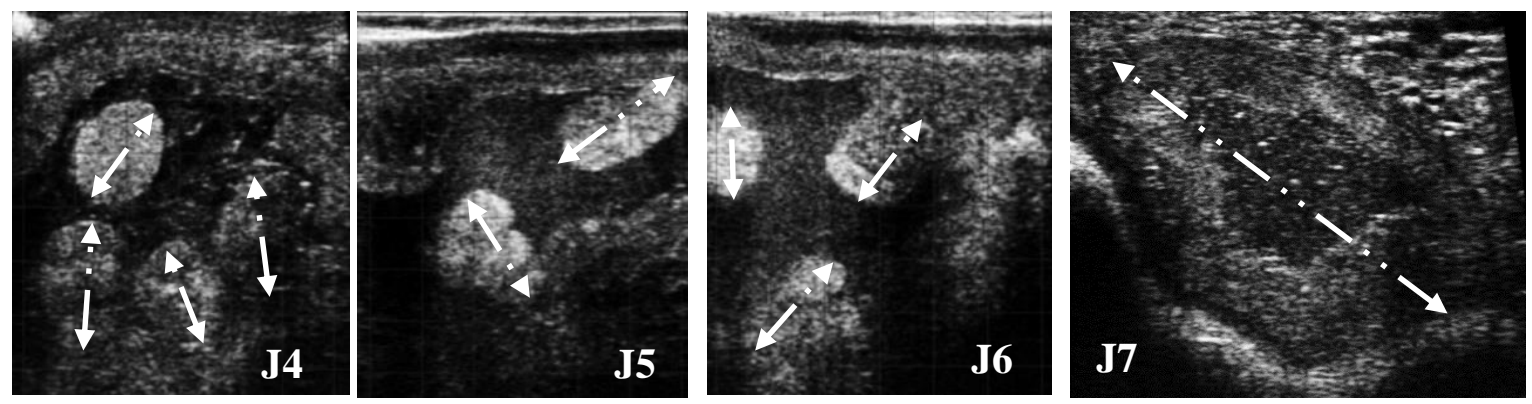

Figure 3 : Images échographiques de la corne utérine de J4 à J7 après parturition.

Tableau 1 : Dynamique de résorption des caroncules utérine après vêlage.

\begin{tabular}{lll}
\hline & \multicolumn{2}{l}{ Diamètre moyen des caroncules utérines (mm) } \\
\hline Jours du postpartum & Primipares & Multipares \\
3 & $27,7 \pm 3,2$ & $25 \pm 6,25$ \\
4 & $25 \pm 3,12$ & $23,43 \pm 4,41$ \\
5 & $18,33 \pm 2,52$ & $22,13 \pm 1,83$ \\
7 & $16,1 \pm 1,27$ & $15,2 \pm 0,55$ \\
9 & $14,06 \pm 0,86$ & $14,53 \pm 0,67$ \\
10 & $10,15 \pm 1,1$ & $10,46 \pm 1,54$ \\
12 & $7,81 \pm 2,2$ & $6,87 \pm 0,88$ \\
13 & - & - \\
\hline
\end{tabular}

a, a : valeurs significativement non différentes entre les lignes 

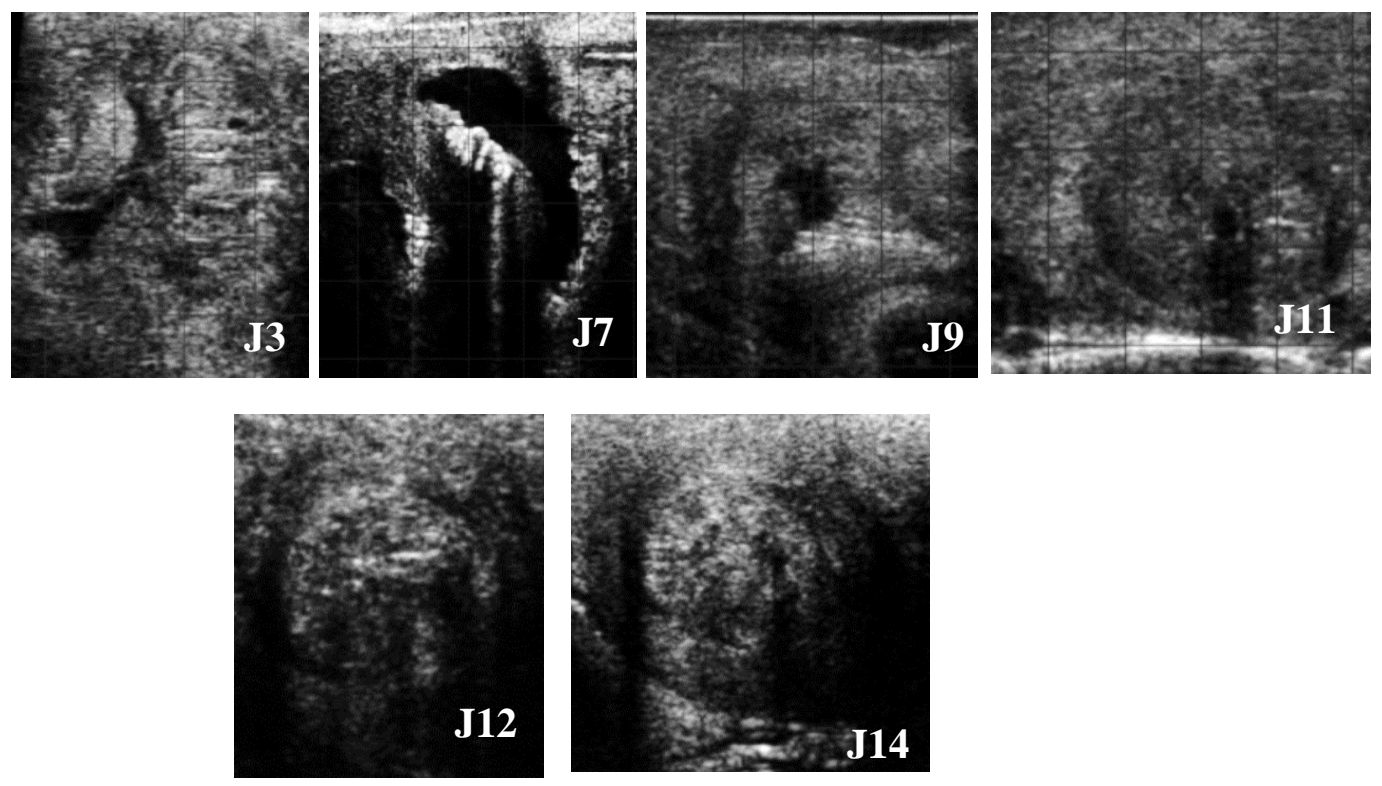

Figure 4 : Images échographiques de l'involution du col utérin après vêlage.
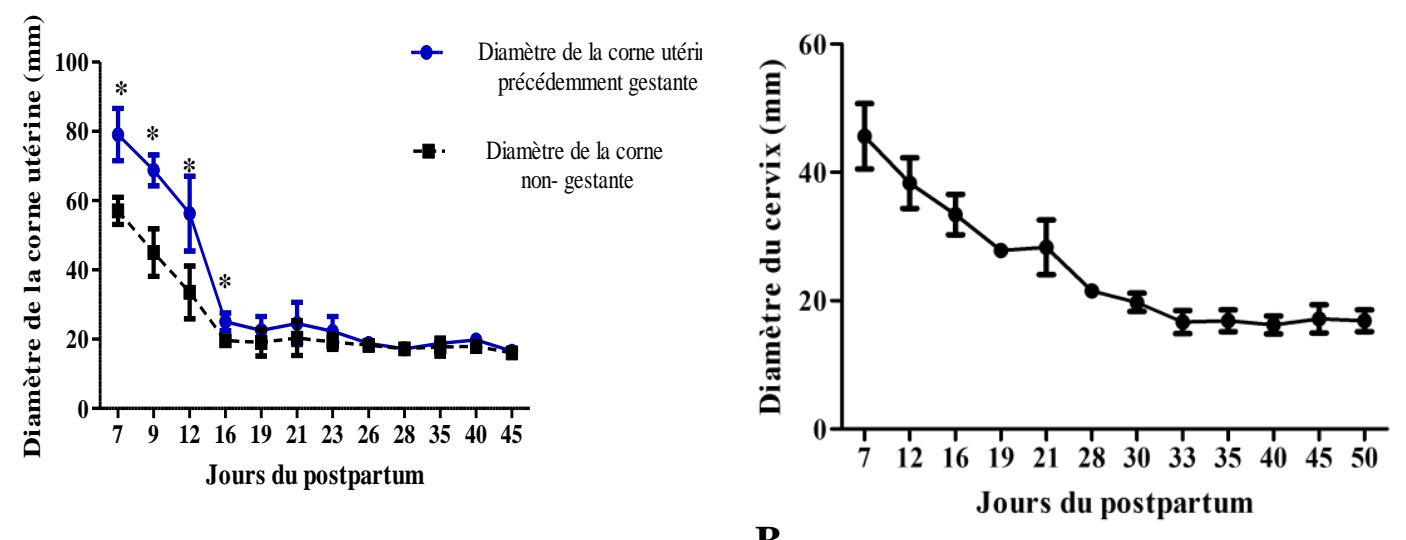

A

B

Figure 5 : Courbes de variation des diamètres des cornes utérines (A) et du cervix (B) au cours du postpartum chez des zébus allaitants.

Tableau 2 : Analyses comparatives de la résorption utérine et cervicale en fonction de la parité.

\begin{tabular}{lcc}
\hline Paramètres évalués & Primipares $(\mathbf{n}=\mathbf{5})$ & Multipares $(\mathbf{n}=\mathbf{5})$ \\
\hline Vitesse de résorption des cornes utérines (mm / jour) & $4,13 \pm 2,88 \mathrm{a}$ & $3,16 \pm 3,19 \mathrm{a}$ \\
Vitesse de résorption du cervix (mm / jour) & $1,39 \pm 0,85 \mathrm{a}$ & $0,85 \pm 0,56 \mathrm{a}$ \\
Délai de l'involution utérine (jours) & $27,6 \pm 1,51 \mathrm{a}$ & $25,6 \pm 2,3 \mathrm{a}$ \\
Délai de l'involution cervicale (jours) & $37,4 \pm 5,17 \mathrm{a}$ & $37,6 \pm 5,46 \mathrm{a}$ \\
Diamètre de la corne utérine après involution (mm) & $17,96 \pm 1,56 \mathrm{a}$ & $18,12 \pm 1,39 \mathrm{a}$ \\
Diamètre du cervix après involution $(\mathrm{mm})$ & $16,66 \pm 1,80 \mathrm{a}$ & $16,24 \pm 1,39 \mathrm{a}$ \\
\hline
\end{tabular}

a, a : valeurs significativement non différentes entre les lignes 

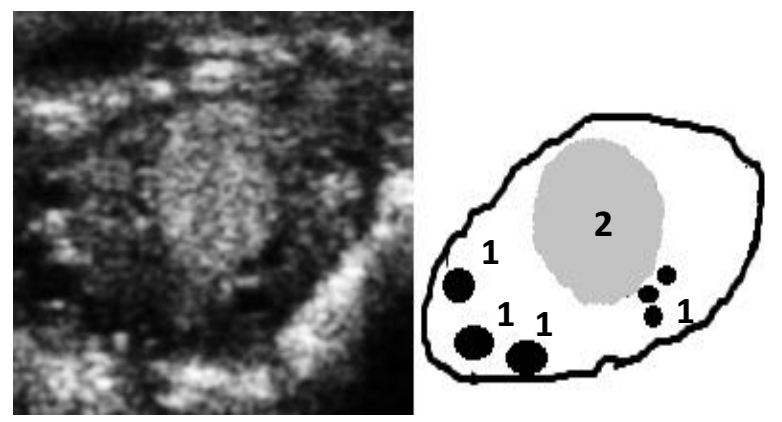

A. Ovaire gauche à $\mathrm{J} 7$ après

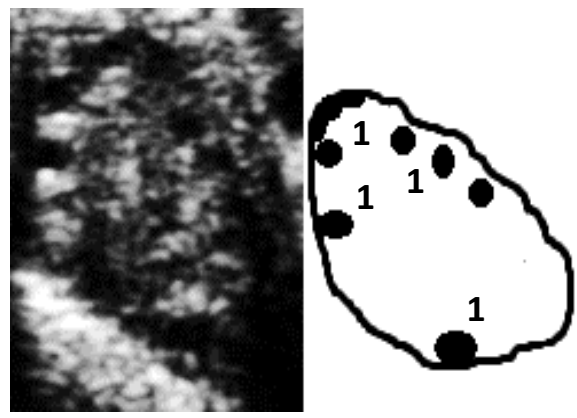

B. Ovaire droit à J7 après

1 - follicule ; 2 - Corps gestatif

Figure 6 : Image échographique des ovaires d'une femelle zébu à J7 postpartum (présence de follicules en évolution).

Tableau 3 : Délais d'apparition des ovulations après vêlage.

\begin{tabular}{|c|c|c|c|}
\hline & Multipares (n=5) & Primipares $(n=5)$ & P value \\
\hline \multirow{5}{*}{$\begin{array}{l}\text { Délai d'apparition de la } \\
\text { première ovulation } \\
\text { (jours) }\end{array}$} & 68 & 73 & - \\
\hline & 57 & 75 & - \\
\hline & 73 & 69 & - \\
\hline & 60 & 74 & - \\
\hline & 65 & 71 & - \\
\hline \multirow[t]{2}{*}{ Délai moyen } & $64,6 \pm 6,34$ & $72,4 \pm 2,4$ & 0,04 \\
\hline & 86 & 93 & - \\
\hline \multirow{4}{*}{$\begin{array}{l}\text { Délai d'apparition de la } \\
\text { deuxième ovulation } \\
\text { (jours) }\end{array}$} & 89 & 85 & - \\
\hline & 84 & 95 & - \\
\hline & 82 & 90 & - \\
\hline & 87 & 94 & - \\
\hline Délai moyen & $85,6 \pm 2,7$ & $91,4 \pm 4,03$ & 0,06 \\
\hline
\end{tabular}

\section{DISCUSSION}

En zone soudano-sahélienne, les rendements des exploitations bovines sont limités par des allongements excessifs des anoestrus post-partum. L'anoestrus postpartum se définit comme la période transitoire au cours de laquelle l'axe hypothalamus-hypophyseovaire-utérus n'est pas en mesure de fonctionner sur le plan physiologique pour permettre une fertilité de la femelle après une mise bas. Cette période est marquée par l'involution de l'utérus (Zhang et al., 2010 ; Sharma et al., 2018) et la reprise des sécrétions des hormones gonadotropes (FSH et LH) par l'hypophysepour relancer le cycle ovarien (Abeygunawardena et Dematawewa, 2004). 
L'involution utérine s'apprécie en routine à travers des examens échographiques ou manuels (ou palpation rectale) de l'utérus. A la différence de l'examen manuel de l'utérus (ou palpation transrectale), l'échographie permet une visualisation en temps réel de la cavité utérine, du cervix et des structures ovariennes (Zhang et al., 2010). Cet avantage serait à l'origine de ses résultats objectifs, parfois complémentaires à la palpation rectale (Heppelmann et al., 2013). Les critères d'une involution utérine complète sont fondés sur la vidange complète de l'utérus, le retour de l'utérus (cervix et cornes) à sa position topographique normale dans la cavité pelvienne, un diamètre utérin inférieur à 2 centimètre, le retour des cornes utérines à une tonicité et à une consistance normale (Sakaguchi et al., 2004 ;Hajurka et al., 2005). Dans la présente étude, l'involution utérine a été caractérisée par deux phases de régressions dont une phase rapide (J7 - J16) qui a connue la résorption complète des caroncules utérines et une phase de régression lente de J17 à J45. Il a été admis chez la vache, l'existence d'une forte concentration de PGF $2 \alpha$ qui peut persister pendant 2 à 3 semaines après vêlage (Mallick et Prakash, 2011). Principalement synthétisée par les caroncules utérines (Deguillaume, 2010), la PGF2 $\alpha$ aurait d'une part, des effets pro-inflammatoire à l'origine d'une vasoconstriction au niveau caronculaire, d'autre part elle stimulerait l'activité électrique du myomètre (Hirsbrunner et al., 2002). Associée à d'autres prostanoïdes comme les leucotriènes B4 (LTB4), l'acide 5 hydroxyeicosa tétraénoïque (HETE), le 15 HETE et la lipoxine B4, la PGF $2 \alpha$ augmenterait l'afflux leucocytaire au niveau utérin et stimulerait l'activité des lymphocytes et des neutrophiles (Deguillaume, 2010). La résorption des caroncules utérines serait une conséquence de la vasoconstriction et de l'activitéphagocytaires des leucocytes (Mann et Lamming, 2000). La régression rapide de l'utérus (cornes utérines et col utérin) serait le résultat des actions stimulatrices de la PGF2 $\alpha$ sur les contractions utérines (Bencharif et al., 2000 ; Yu et al., 2016). Au cours de cette période (J7 - J30), l'ovaire connaît une dynamique folliculaire active, mais les follicules n'arrivent pas à maturité et donc n'ovulent pas. Cette observation a été rapportée par Sakaguchi et al. (2004).

Dans la présente étude, le délai moyen de l'involution utérine a été de 26,6 $\pm 2,11$ jours. Les femelles primipares semblent présenter une involution utérine tardive comparativement aux femelles multipares. De même, les premières et deuxièmes ovulations postpartum sont apparues précocement chez les femelles zébu multipares comparativement aux primipares. De plus, les manifestations d'œstrus ont été observées en première ovulation et deuxième ovulation dans respectivement $2 / 5$ et $4 / 5$ des femelles multipares. Chez les femelles primipares, les manifestations d'œstrus sont apparues au cours de la deuxième ovulation chez $2 / 5$ des femelles. L'effet et le mécanisme d'action de la parité sur la reprise de la cyclicité restent encore imprécis et controversés (Zhang et al., 2010 ; Sharma et al., 2018 ; Canadas et al., 2019). Nos résultats concordent avec les données de la littérature (Zhang et al., 2010 ; Kara et al., 2013). Chez la race taurine d'Europe et d'Amérique, les primipares présentent des délais d'involution utérine ou un intervalle parturition - première ovulation plus longs que les multipares (Sharma et al., 2018). Ces derniers expliquent que le mécanisme d'action de la parité passe par la balance énergétique de l'animal. Aussi, chez les primipares en croissance, la fréquence des pulses de LH au cours du post-partum est lente (Nogueira et al., 2013 ; Sharma et al., 2018). De plus, chez la vache Brahman, les primipares développent $34 \%$ en plus de vagues folliculaires que les multipares (Ramirez, 2006). Ce qui pourrait prolonger l'anoestrus postpartum d'une à quatre semaines (Rekwot et al., 2000). D'autres auteurs rapportent un effet 
positif de la parité sur la durée de l'anoestrus postpartum (Hajurka et al., 2005). Ceci pourrait s'expliquer par la différence de la fréquence des ovulations des vagues folliculaires successives entre les primipares et les multipares. Selon Sakaguchi et al. (2004), les femelles laitières primipares présentent des fréquences d'ovulation à la première vague et deuxième vague folliculaires de $50 \%$ et $50 \%$ respectivement contre $33 \%$ et $35 \%$ chez les multipares.

\section{Conclusion}

Sous les conditions tropicales, la femelle zébu allaitante manifeste une involution utérine complète qui précède la reprise de l'activité ovarienne et la manifestation œstrale. La durée de l'intervalle varie largement avec un minimum de 38 jours et un maximum de 62 jours.

\section{CONFLIT D'INTERETS}

Les auteurs déclarent ne pas avoir de conflit d'intérêts.

\section{CONTRIBUTIONS DES AUTEURS}

MZ a défini le sujet de recherche et superviser les travaux. ATY et MZ ont réalisé les analyses statistiques et rédiger le manuscrit. ATY, BT, BHK ont participé à la collecte des données.

\section{REMERCIEMENTS}

Les auteurs remercient la Fondation International pour la Science (FIS) pour leur appui technique et financier dans la réalisation de la présente étude. Ils souhaitent que l'Agence Internationale pour l'Energie Atomique (AIEA) trouve à travers cet article, l'expression de leur reconnaissance pour les équipements offerts.

\section{REFERENCES}

Abeygunawardena H, Dematawewa CMB. 2004. Pre-pubertal and postpartum anestrus in tropical Zebu cattle. Anim.
Reprod. $\quad$ Sci., $\quad$ 82-83: $\quad 373-387$. DOI:https://doi.org/10.1016/j.anireprosci .2004.05.006

Bencharif D, Tainturier D, Slama H, Bruyas JF, Battut I, Fieni F. 2000. Prostaglandines et post-partum chez la vache. Revue Med. Vet.,151(5): 401-408.

Canadas ER, Lonergan P, Butler ST. 2019. Effect of equine chorionic gonadotropin administration on day 8 postpartum on ovarian follicular development, uterine health and uterine involution in lactating dairy cows. Theriogenology, 123 (1): 5461.DOI:

https://doi.org/10.1016/j.theriogenology. 2018.09.022

Deguillaume L. 2010. L'inflammation génitale post-partum de la vache. Thèse de Doctorat, Institut des Sciences et Industries du Vivant et de l'Environnement, AgroParisTech, Paris,p. 206.

FAO. 2018. Le développement durable de l'élevage africain: approche «Une seule santé » au Burkina Faso. Rome, FAO, p.104.

Hajurka J, Macak V, Hura V. 2005. Influence of health status of reproductive organs on uterine involution in dairy cows. Bull. Vet. Inst. Pulawy, 49(1): 53-58.

Heppelmann M, Brömmling A, Weinert M, Piechotta M, Wrenzycki C, Bollwein H, 2013. Effect of postpartum suppression of ovulation on uterine involution in dairy cows. Theriogenology, 80(5): $519-25$. DOI: https://doi.org/10.1016/j.theriogenology. 2013.05.017

Hirsbrunner G, Knutti B, Liu I, Küpfer U, Scholtysik G, Steiner A. 2002. An in vivo study on spontaneous myometrial contractility in the cow during estrus and diestrus. Anim. Reprod.Sci.,70(3-4): 17180. DOI: https://doi.org/10.1016/S03784320(01)00198-1 
Kara N, Bounechada M, Chaib B C. 2013. Effect of body condition score and parity on resumption of post-partum ovarian activity in Montbeliard dairy cows under semi-arid area of Algeria. J Anim. Sci. $A d v ., \quad 4(4): \quad 787-96 . \quad$ DOI: 10.5455/jasa.20130219032046

Mallick S, Prakash BS. 2011. Effects of supplementation of Tinospora cordifolia to crossbred cows peripartum. Anim. Reprod. Sci., 123(1-2): 5-13. DOI: https://doi.org/10.1016/j.anireprosci.201 0.11 .008

Mann GE, Lamming GE. 2000. The role of sub-optimal preovulatory oestradiol secretion in the aetiology of premature luteolysis during the short oestrus cycle in the cow. Anim. Reprod. Sci., 64(3-4): 171-180.

DOI:

https://doi.org/10.1016/S03784320(00)00205-0

Marichatou H, Issa M, Hamadou I, Assane M, Semita C. 2010. Efficacité de la synchronisation des chaleurs et l'insémination artificielle chez les bovins Azawak : intérêt du profil de progestérone. Tropicultura, 28: 161-167.

MRA. 2010. Politique Nationale de Développement Durable de l'Elevage au Burkina Faso, 2010-2025, Ouagadougou, p.45.

MRA. 2011. Annuaires statistiques du secteur de l'élevage, Burkina Faso, Ouagadougou, p. 151.

Mwai O, Hanotte O, Kwon YJ, Cho S. 2015. African indigenous cattle: unique genetic resources in a rapidly changing world. Asian Austral J. Anim.,28(7): 911-921. DOI: 10.5713/ajas.15.0002R

Nogueira E, Batista Dayanna do N S, Filho L C C da C, Dias A M, Silva J C B, Ítavo L C V, 2014. Pregnancy rate in lactating Bos indicus cows subjected to fixed-time artificial insemination and treated with different follicular growth inducers. $R$. Bras. Zootec., 43 (7): 358 - 362. DOI :
https://doi.org/10.1590/S151635982014000700003

Pierson RA, Ginther OJ. 1988. Ultrasonic imagingof the ovaries and uterus in cattle. Theriogenology, 29(1): 21-37. DOI: https://doi.org/10.1016/0093691X(88)90029-5

Pitala W, Zongo M, Boly H, Sawadogo L, Leroy P, Beckers JF, GBeassor M. 2012. Étude de l'oestrus et de la fertilité après un traitement de maîtrise des cycles chez les femelles zébus. Int. J. Biol. Chem. Sci., 6(1): 257-263. DOI: https://doi.org/10.4314/ijbcs.v6i1.22

Ramirez J. 2006. Effect of once-daily suckling and parity on follicular dynamics in postpartum Brahman cows. Memoire de Master, Texas A\&M University, p.63.

Rekwot PI, Ogwu D, Sekoni VO, Oyedipe EO. 2000. Serum progesterone profiles of zebu cattle (Bos indicus) in relationship to conception and repeat breeding after artificial insemination. Anim. Reprod. Sci., 63(1-2): 41-51. DOI: https://doi.org/10.1016/S03784320(00)00167-6

Sakaguchi M, Sasamoto Y, Suzuki T, Takahashi Y, Yamada Y. 2004. Postpartum ovarian follicular dynamics and estrous activity in lactating dairy cows. $J$. Dairy Sci., 87(7): 2114-2121. DOI: https://doi.org/10.3168/jds.S00220302(04)70030-2

Savio JD, Boland MP, Roche JF. 1990. Development of dominant follicles and length of ovarian cycles in post-partum dairy cows. J. Reprod. Fert., 88 (2): 581591.

DOI: https://doi.org/10.1530/jrf.0.0880581

Seme K, Pitala W, Kulo AE, Talaka E, Gabiam KB, Djabangou P, Kotoe MD, Gbeassor M. 2017. Etude de l'œstrus des femelles bovins laitiers soumises au protocole Prid® Delta au Sud-Togo. Int. J. Biol. Chem. Sci., 11(3): 958-966. DOI: https://dx.doi.org/10.4314/ijbcs.v11i3.3 
Sharma A, Singh M, Sharma A, Kumar P. 2018. Effect of BCS and parity on uterine involution, ovarian rebound and various fertility parameters in postpartum dairy cows. Indian J. Anim. Sci., 88(5): 526 529. DOI: 10.14302/issn.25751212.jvhc-18-2447

Touré A, Antoine-Moussiaux N, Geda F, Kouriba A, Traoré D, Traoré B, Leroy P, Moula N. 2019. Phenotypic parameters affecting reproduction and production performances of dairy cattle in peri-urban of Bamako, Mali. Veterinary World, 12(6): 817-822. DOI: 10.14202/vetworld.2019.817-82

Toribio RE, Molina JR, Forsberg M, Kindahl H, Edqvist LE, 1995. Effects of calf removal at parturition on postpartum ovarian activity in Zebu (Bos indicus) cows in the humid tropics. Acta. Vet. Scand., 36(3): 343-352.

Yahaya ZI, Dayo G-K, Maman M, Issa M, Marichatou H. 2019. Caractérisation morphobiométrique du zébu Djelli du
Niger. Int. J. Biol. Chem. Sci.,13(2): 727744.

DOI:

https://dx.doi.org/10.4314/ijbcs.v13i2.13

Yu G-M, Baic J-H, Liuc Y, Maeda T, Zenga SM. 2016. A weekly postpartum PGF2a protocol enhances uterine health in dairy cows. Reprod Biol., 16(4) : 295-299. DOI: 10.1016/j.repbio.2016.10.006

Zampaligré N, Savadogo I, Sangare M. 2019. Analyses des paramètres démographiques et zootechniques du cheptel bovin des élevages péri-urbains laitiers de la ville de Bobo-Dioulasso à l'Ouest du Burkina Faso. Int. J. Biol. Chem. Sci., 13 (1): 441451.

DOI: https://dx.doi.org/10.4314/ijbcs.v13i1.35

Zhang J, Deng LX, Zhang HL, Hua GH, Han L, Zhu Y, Meng XJ, Yang L G. 2010. Effects of parity on uterine involution and resumption of ovarian activities in postpartum Holstein dairy cows. J Dairy Sci., 93(5): 1979-1986. DOI: https://doi.org/10.3168/jds.2009-2626. 